Original

\title{
A comparative scanning electron microscopic study of the effect of three different rotary instruments on smear layer formation
}

\author{
Shahriar Shahi ${ }^{1)}$, Hamid R. Yavari ${ }^{1)}$, Saeed Rahimi ${ }^{1)}$, Mohammad F. Reyhani ${ }^{1)}$, \\ Zahra Kamarroosta ${ }^{2)}$ and Majid Abdolrahimi ${ }^{2)}$ \\ ${ }^{1)}$ Department of Endodontics, Tabriz Dental School, Tabriz University (Medical Sciences), Tabriz, Iran \\ ${ }^{2)}$ Private practice, Tabriz, Iran
}

(Received 22 May and accepted 20 November 2008)

\begin{abstract}
The aim of this study was to evaluate the effect of RaCe, FlexMaster and ProFile rotary instruments on smear layer formation by scanning electron microscopy. Eighty-four caries-free freshly extracted human single-rooted teeth were selected and divided into three groups, each containing 28 teeth. The teeth were instrumented with rotary instruments sequentially: Group A: ProFile Rotary Instruments; Group B: FlexMaster Rotary Instruments; and Group C: RaCe Rotary Instruments. Instrumentation was performed by the crown-down method and according to the manufacturer's instructions. The specimens were then examined with SEM according to Hülsmann's classification. One-way ANOVA and a post hoc Tukey test were used for statistical analysis. The results showed that there were no statistically significant differences among the three groups in the coronal third $(P=0.39)$, but at the apical and middle thirds there were statistically significant differences between the RaCe group and the other groups $(P<0.05)$. Smear layer in the RaCe group was less than that in the ProFile and FlexMaster groups, but the difference between the ProFile group and FlexMaster group was not statistically significant $(P>0.05)$. It was concluded that RaCe Rotary Instruments produce less smear layer than FlexMaster and ProFile Rotary Instruments. (J Oral Sci 51, 55-60, 2009)
\end{abstract}

Correspondence to Dr. Shahriar Shahi, Department of Endodontics, Tabriz Dental School, Tabriz University (Medical Sciences), Golgasht Street, 5166614713, Tabriz, Iran

Tel: +98-914-314-2971

Fax: +98-411-334-6977

E-mail: shahriar_shahi@hotmail.com
Keywords: Smear layer; rotary instruments; scanning electron microscope; root canal preparation.

\section{Introduction}

The main goal of root canal instrumentation is to clean and shape the root canal system (1). Studies have shown that current methods of cleaning and shaping root canals produce a smear layer that covers the instrumented walls (2-5). This layer contains inorganic and organic substances, including fragments of odontoblastic processes, microorganisms, and necrotic materials (5). According to Mader et al, the smear layer consists of a superficial layer on the surface of the canal wall, approximately 1 to $2 \mu \mathrm{m}$ in thickness, and a deeper layer is packed into the dentinal tubules to a depth of up to $40 \mu \mathrm{m}$ (3). The components of the smear layer can be forced into the dentinal tubules to varying distances (4). This can occur as a result of the linear movement and rotation of instruments and because of capillary action generated between the dentinal tubules and the smear material (4). The smear layer covers the instrumented walls and may prevent close adaptation between root canal filling materials and the root canal walls (6). However, instruments for narrow canals with radial lands have a larger contact area with the canal wall, which increases friction and torque and thereby fracture risk $(7,8)$.

Today, many rotary nickel-titanium files are available in various geometric designs. There are passive instruments with radial lands, such as ProFile (Dentsply, Maillefer, Ballaigues, Switzerland). A radial land is a flat area located directly behind the cutting edge (9). On the other hand, 
there are also active instruments, such as FlexMaster (Vereinigte Dentalwerke, München, Germany) and RaCe (FKG Dentaire, La Chaux-de-fonds, Switzerland). ProFile rotary reamers have three flat radial lands with a negative rake angle and a constant helix angle. Studies have demonstrated that ProFile reamers properly maintain the original canal axis and a good three-dimensional form $(10,11)$.

$\mathrm{RaCe}$ instruments $(\mathrm{RaCe}=$ "reamer with alternating cutting edges") have a triangular cross-sectional design with sharp cutting edges, with the exception of 0.02 taper \#20 files, which have a square cross-section. These sharp cutting edges would result in efficient chip dislodgment (12).

Schirrmeister et al. reported that $\mathrm{RaCe}$ rotary files are safe and more effective compared to FlexMaster, ProFile and ProTaper files (13). Schäfer and Lohmann reported that K-Flexofiles allowed significantly better canal cleaning than FlexMaster instruments, while FlexMaster instruments maintained the original curvature much better (14). Schäfer and Vlassis concluded that $\mathrm{RaCe}$ instruments resulted in relatively good cleaning and maintained the original curvature significantly better than ProTaper instruments did (15). Zand et al. reported that FlexMaster rotary instruments left significantly less debris and smear layer than RaCe rotary and Ni-Ti flex K-file hand instruments (16). On the other hand, Zarrabi et al. concluded that $\mathrm{RaCe}$ system extrudes less debris than the manual technique and FlexMaster system (17). At present, more information about the effectiveness of RaCe rotary instruments with regard to smear layer formation is needed, and the efficacy of $\mathrm{RaCe}$ instruments should be compared with that of other differently designed nickel-titanium rotary instruments such as FlexMaster and ProFile systems. Thus, the purpose of this study was to evaluate the effect of RaCe, FlexMaster and ProFile rotary instruments on smear layer formation by scanning electron microscopy.

\section{Materials and Methods}

Eighty-four human single-rooted teeth were used in this study. Preoperative radiographs showed absence of multiple canals, calcification, or severe apical curvatures. The teeth had been extracted for periodontal reasons. The teeth were kept in $0.5 \%$ Chloramine T solution (Merk, Darmsladt, Germany) at $4{ }^{\circ} \mathrm{C}$ until use. The degree of canal curvature was determined using the Schneider's method (18) and the teeth with curvatures less than $10^{\circ}$ and length of 21-22 mm were selected. Straight-line access cavities were obtained in all the specimens and then the teeth were randomly divided into three experimental groups, each containing 28 teeth, as follows:
Group A: ProFile rotary instruments (Dentsply, Maillefer, Ballaigues, Switzerland); Group B: FlexMaster rotary instruments (Vereinigte Dentalwerke, M?nchen, Germany); and Group C: RaCe rotary instruments (FKG Dentaire, La Chaux-de-fonds, Switzerland).

One investigator performed all the steps of root canal instrumentation. The working lengths (WL) were established within $1 \mathrm{~mm}$ of the apical foramina using file \#20 (K-file, Maillefer, Ballaigues, Switzerland). Roots in which the initial instrumentation to the apical foramina could be performed larger than \#20 K-file were not used in this study. All the root canals were prepared with instruments up to \#40 and a new set of instruments was used for each canal. The instrumentation was performed in slight continuous, in-and-out movements and the instruments were never forced apically. Copious irrigation was performed after each instrumentation via a $5-\mathrm{mL}$ SUPA syringe (Tehran, Iran), which delivered $4 \mathrm{~mL}$ of sterile saline solution ( $0.9 \%$ sodium chloride) through a 27-gauge needle (Iran Needle, Tehran, Iran). The root canals were kept flooded with the irrigation solution throughout the entire instrumentation procedure. The instrumentation sequences used in the present study were as follows:

ProFile group: These instruments were set into rotational speed $(300 \mathrm{rpm})$ with an 8:1 reduction handpiece powered by torque limited electric motor (TCM Motor 3000 Novage, Konstanz, Germany). The canals were instrumented to two-thirds of the working length using ProFile \#35/06 taper, \#30/06 taper, \#30/04 taper, and \#25/04 taper instruments. This was followed by the sequential use of the ProFile \#25/02 taper, \#30/04 taper, \#30/06 taper, 35/02 taper and \#40/02 taper instruments to the final working length with the application of gentle pressure.

FlexMaster group: All the procedures were the same as described for ProFile group except for fact that FlexMaster \#40/06 taper was used in this group and all the instrumentations were similar to the previous group.

RaCe group: All the procedures were similar to the two other groups except for the fact that $\mathrm{RaCe} \# 40 / 0.1$ taper and \#35/0.08 taper were used in this group and the instrumentation series was similar to the other two groups.

On completion of instrumentation, each canal was flushed with $5 \mathrm{ml}$ of sterile saline solution. The canals were thoroughly dried with sterile paper points (Aria Dent, Tehran, Iran). The teeth were decoronated at the cementoenamel junction (CEJ). The teeth were then split in half after two parallel longitudinal grooves were made with a slow-speed diamond disk on the outer surface of the roots. The root was split with a plastic instrument used as a chisel to prevent contamination of the canals during the 
separation process.

Two halves of each root were used to look down onto the smear layer to evaluate the extent of this layer on the canal wall surface in the apical, middle, and coronal thirds. The root halves were dehydrated in a desiccator for $48 \mathrm{~h}$, coated with a thin $(200 \AA)$ palladium-gold film and viewed with a scanning electron microscope (SEM) (JSM 6320F; Japanese Electron Optics Laboratory, Tokyo, Japan). After the central beam of the SEM was directed to the apical, middle and coronal thirds of the root canal wall under $\times 45$ magnification, five areas of each specimen (10 areas for each root) were randomly selected on the screen. In each selected area, the magnification was increased to $\times 1,500$ $-\times 3,000$ and a transparent grid was placed on the SEM screen. The cleanliness of the root canal walls was evaluated on 10 pre-selected squares of the grid with the following five-score system (19);

Score 1: No smear layer; dentinal tubules open

Score 2: Small amount of smear layer; some dentinal tubules open

Score 3: Homogenous smear layer covering the root canal wall; only few dentinal tubules open

Score 4: Complete root canal wall covered by a homogenous smear layer; no open dentinal tubules Score 5: Heavy, nonhomogenous smear layer covering the complete root canal wall.

The scoring procedure was performed by a second operator who was blinded to the individual who had prepared the root canals, and the coded specimens, and the device used for root canal preparation. Statistical analysis was performed using one-way analysis of variance (ANOVA) followed by a post hoc Tukey test. Statistical significance was defined as $P<0.05$.

\section{Results}

In the three instrumentation groups, there were differences in the general surface appearance of the smear layer on the root canal walls (Fig. 1). Completely cleaned root canals were not found in any of the groups.

Statistical analysis showed that there were no significant differences among the 3 experimental groups in the coronal third $(P=0.39)$. However, in the middle and apical thirds, there were statistically significant differences among the groups. In the middle third, the differences between the RaCe group and the ProFile group $(P=0.04)$ and between the RaCe group and the FlexMaster group $(P=0.002)$ were

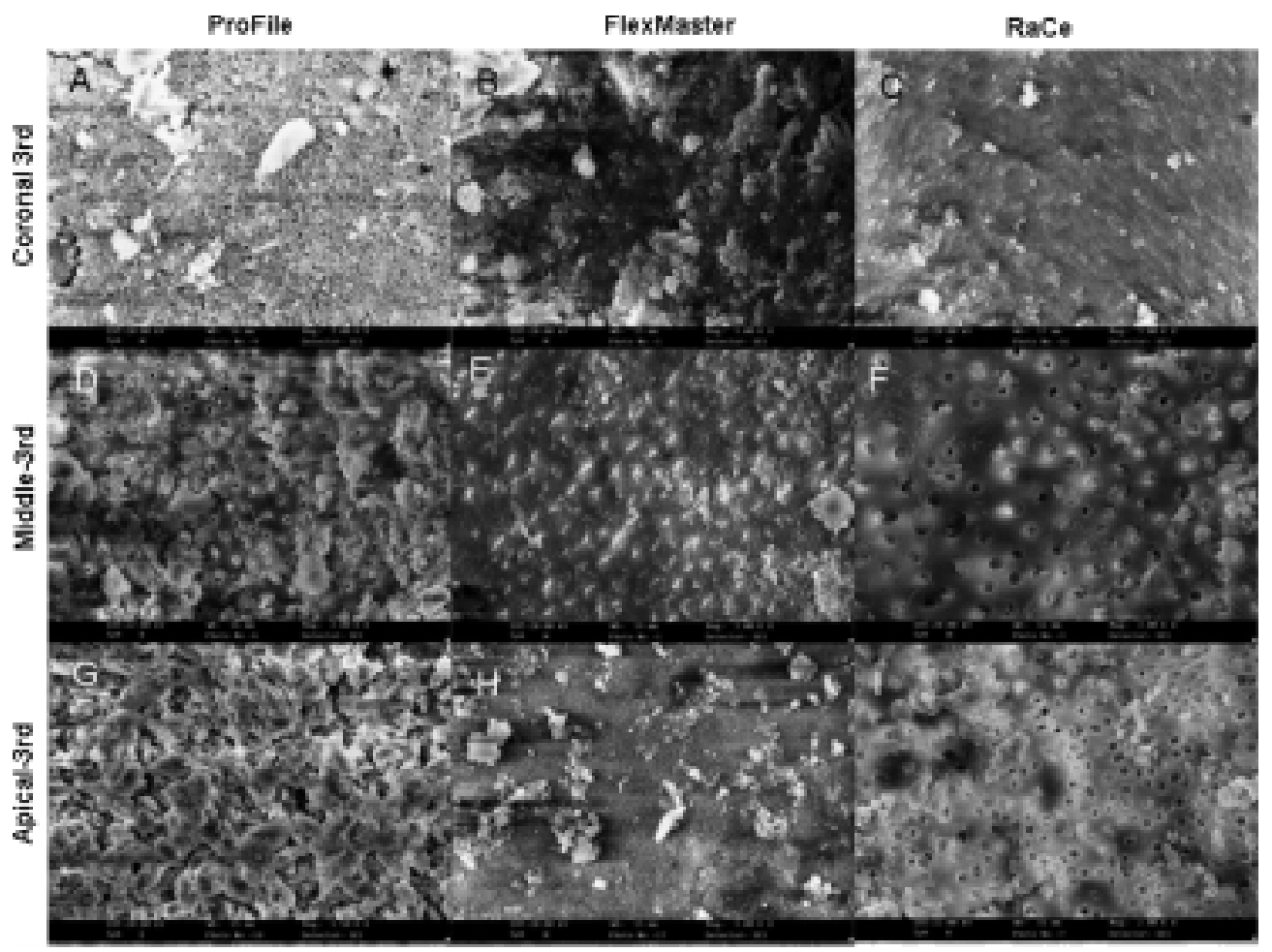

Fig. 1 SEM photomicrographs (magnification $\times 2,000$ and $\times 3,000$ ) of canal walls prepared with ProFile, FlexMaster and RaCe rotary instruments. Smear layer formation can be observed in all the systems under study and open dentinal tubules cannot be seen in the coronal third. RaCe instruments have produced cleaner canal walls in the middle and apical thirds compared to ProFile and FlexMaster instruments. 
statistically significant. On the other hand, in the apical third, the differences between the $\mathrm{RaCe}$ group and the ProFile group $(P<0.0005)$ and between the RaCe group and the FlexMaster group $(P<0.0005)$ were statistically significant (Fig. 2).

According to the results of one-way ANOVA, there were no significant differences in the means of smear layer formation in the entire length of the canals between the experimental groups $(P=0.003)$. The results of the post hoc Tukey test revealed significant differences between the $\mathrm{RaCe}$ and FlexMaster groups in this regard $(P=0.002)$. The differences in the means of smear layer production between the other groups were not statistically significant $(P>0.05)$ (Fig. 3).

\section{Discussion}

In the present in vitro study, only normal saline was used as the irrigant so that the cleaning efficacy of the three canal

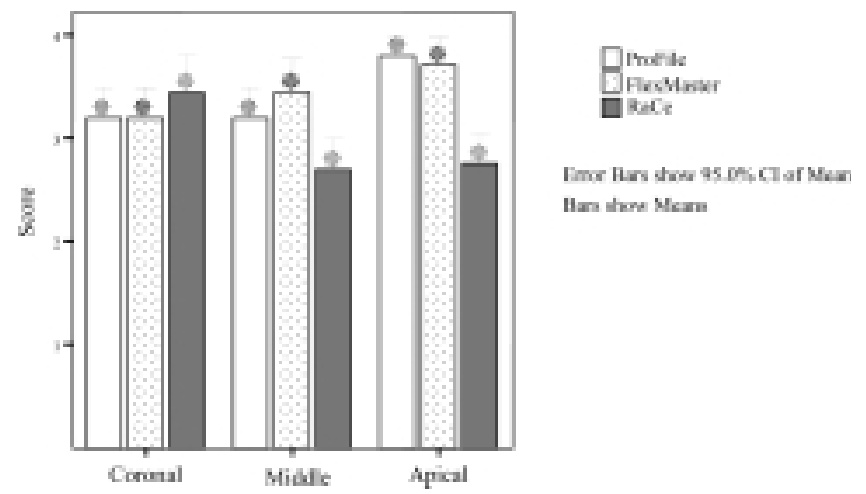

Fig. 2 Error bar diagram of the comparison of smear layer score means. Comparison of the different portion of the canals.

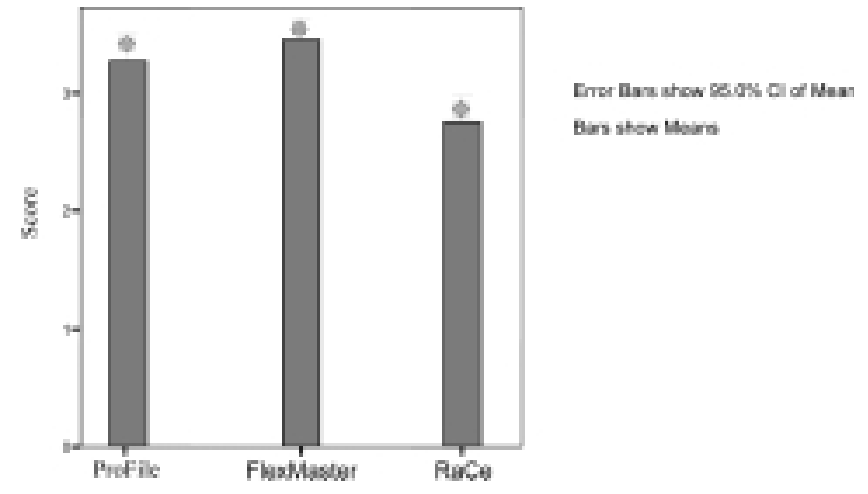

Fig. 3 Error bar diagram of the comparison of smear layer score means. Comparison in the entire length of the canals. preparation methods could be evaluated and compared without the intervention of confounding factors. It should be pointed out the cleaning efficacy of the three root canal preparation methods used in the present study might have been improved with the combined use of EDTA and high concentrations of $\mathrm{NaOCl}$.

An important factor considered in the present study was the inclusion of caries-free single-rooted teeth in the study design, eliminating the possible role of caries in smear layer formation. On the other hand, the crowns of the teeth were not removed before instrumentation so that the clinical situation could be simulated as closely as possible. Another important consideration in such studies is the blindness of the individual evaluating the samples to the procedure used in the study so that he/she would not be able to discover the codes used and could therefore evaluate the samples without any prejudgment. To this end, the samples were submitted to the SEM operator along with the previous codes as follows: Group A, ProFile; Group B, FlexMaster; Group C, RaCe; the operator then received a full explanation and chose different kinds of codes without the researchers having any knowledge about the relationship between the previous and new codes.

In the present study, some relatively uninstrumented areas were found in the coronal, middle and apical thirds in all three preparation methods, which is consistent with the results of other studies, such as those carried out by Hülsmann (19) and Schäfer (14). In the current study, there were no statistically significant differences in the frequency distribution of smear layer formed by the three different types of instruments in the coronal thirds and the smear layer mostly observed belonged to score 4 , which is consistent with the results of a study carried out by Schäfer in 2002. The abovementioned study revealed that FlexMaster files leave more smear layer compared to hand instruments, especially in the coronal third (14).

In the present study, statistically significant differences were observed in smear layer formation between the three experimental groups: less grade 2 smear layer was formed in the RaCe group, which is not consistent with the results of a study carried out by Schäfer in 2004 (15). They compared the smear layer formation in $\mathrm{RaCe}$ and ProTaper rotary instruments, and observed that $\mathrm{RaCe}$ rotary instruments left less smear layer, although the differences were not statistically significant. In their study, mandibular and maxillary molars had been used. The roots had curvatures ranging between 25 and 45 degrees and 2.5\% $\mathrm{NaOCl}$ was used as the irrigant for canal preparation. As $\mathrm{NaOCl}$ can dissolve organic components of the smear layer it might have influenced the results of the study. On the other hand, RC Prep had been used as a lubricating 
agent in that study, which has EDTA as a major ingredient with the ability to dissolve salts. Smear layer contains both organic and inorganic ingredients, and the use of RC Prep can influence the results of the study.

Statistically significant differences were observed in smear layer formation in the apical third of the root canals among the three experimental groups. The least amount of smear layer was observed in the RaCe group with a $35 \%$ frequency of score 2 , which is consistent with the results of a study carried out by Schäfer and Vlassis (15) in 2004, indicating the better cleaning efficacy of RaCe rotary instruments. In the apical third of the roots, the greatest amount of smear layer was observed in the ProFile group with an $80 \%$ frequency of score 4 . The results of a study carried out by Versümer et al. in 2002 indicated that ProFile instruments leave the greatest amount of smear layer (20).

Two-by-two comparison of the instruments in the present study in the coronal, middle and apical thirds of the root canals did not demonstrate any significant differences between FlexMaster (without radial lands) and ProFile (with radial lands) instruments and the smear layer grades were similar. According to the results of the present study, it seems that radial lands of instruments have less influence on producing smear layer; however, further studies should be carried out to confirm this hypothesis. Comparison of $\mathrm{RaCe}$ and FlexMaster instruments in the coronal third did not reveal any significant differences and score 4 smear layer was observed with higher frequency in the FlexMaster group. There were significant differences in the middle third between the RaCe and FlexMaster groups and less smear layer with higher score 2 frequency was observed in the RaCe group. The same results were obtained in the apical third, which might be explained by the fact that RaCe instruments have wider furrows, sharp cutting edges and active cutting blades, leading to an increased cutting capacity, which facilitates the movement of debris in the coronal direction leaving less smear layer in the apical third. However, FlexMaster instruments are locked on the coronal walls and have narrow furrows causing the file and debris to move toward the apical third. Jeon et al have reported that nickel-titanium rotary files with active cutting blades increase root canal cleanliness by removing smear layer better than instruments with radial lands, which seem to burnish the smear layer (21). On the other hand, Zand et al. concluded that FlexMaster files leave less smear layer and debris in comparison to RaCe instruments (16), which does not coincide with our results. Zand et al. used $\mathrm{NaOCl}$ as an irrigating solution and evaluated smear layer and debris separately. $\mathrm{NaOCl}$ might have influenced the results of that study by dissolving organic components of smear layer. It should be emphasized that it is difficult to distinguish smear layer from debris.

It appears that $\mathrm{RaCe}$ instruments leave smear layer with lower grades in comparison to FlexMaster and ProFile instruments. The lower amount of the smear layer was more noticeable in the middle and apical thirds but smear layer in the coronal third was more abundant; however, the differences were not significant. In general, $\mathrm{RaCe}$ instruments left less smear layer in the present study.

Considering the limitations of the present study and its results and the absence of sodium hypochlorite for irrigation and chelating agents during instrumentation, it is suggested that further studies be carried out with the use of sodium hypochlorite and EDTA.

\section{Acknowledgments}

The authors would like to extend their appreciation to the Office of Vice Chancellor for Research, Tabriz University (Medical Sciences), for supporting this research financially.

\section{References}

1. Schilder H (1974) Cleaning and shaping the root canal. Dent Clin North Am 18, 269-296.

2. McComb D, Smith DC (1975) A preliminary scanning electron microscopic study of root canals after endodontic procedures. J Endod 1, 238-242.

3. Mader CL, Baumgartner JC, Peters DD (1984) Scanning electron microscopic investigation of the smeared layer on root canal walls. J Endod 10, 477483.

4. Cengiz T, Aktener BO, Pikin B (1990) The effect of dentinal tubule orientation on the removal of smear layer by root canal irrigants. A scanning electron microscopic study. Int Endod J 23, 163-171.

5. Pashly DH (1992) Smear layer: overview of structure and function. Proc Finn Dent Soc 88, Suppl 1, 215224.

6. Toraninejad M, Handysides R, Khademi AA, Bakland LK (2002) Clinacal implications of the smear layer in endodontics: a review. Oral Surg Oral Med Oral Pathol Oral Radiol Endod 94, 658666.

7. Machtou P, Ruddle CJ (2004) Advancements in the design of endodontic instruments for root canal preparation. Alpha Omegan 97, 8-15.

8. Blum JY, Machtou P, Michallef JP (1999) Location of contact areas on rotary Profile instruments in relationship to the forces developed during mechanical preparation on extracted teeth. Int Endod J 32, 108-114. 
9. Koch K, Brave D (2002) Real World Endo: design features of rotary files and how they affect clinical performance. Oral Health 92, 39-49.

10. Kum KY, Spängberg L, Cha BY, Il-Young J, SeungJong L, Chan-Young L (2000) Shaping ability of three ProFile rotary instrumentation techniques in simulated resin root canals. J Endod 26, 719-723.

11. Bryant ST, Thompson SA, al-Omari MA, Dummer PM (1998) Shaping ability of ProFile rotary nikeltitanium instruments with ISO sized tips in simulated root canals: Part 1. Int Endod J 31, 275-281.

12. Bergmans E, Van Cleynenbreugel J, Wevers M, Lambrechts P (2001) Mechanical root canal preparation with NiTi rotary instruments: rationale, performance and safety. Status report for the American Journal of dentistry. Am J Dent 14, 324333.

13. Schirrmeister JF, Strohl C, Altenburger MJ, Wrbas KT, Hellwig E (2006) Shaping ability and safety of five different rotary nickel-titanium instruments compared with stainless steel hand instrumentation in simulated curved root canals. Oral Surg Oral Med Oral Pathol Oral Radiol Endod 101, 807-813.

14. Schäfer E, Lohmann D (2002) Efficiency of rotary nickel-titanium FlexMaster instruments compared with stainless steel hand K-Flexofile - Part 2. Cleaning effectiveness and instrumentation results in severely curved root canals of extracted teeth. Int Endod J 35, 514-521.

15. Schäfer E, Vlassis M (2004) Comparative investigation of two rotary nickel-titanium instruments: ProTaper versus RaCe. Part 2. Cleaning effectiveness and shaping ability in severely curved root canals of extracted teeth. Int Endod J 37, 239248.

16. Zand V, Bidar M, Ghaziani P, Rahimi S, Shahi S (2007) A comparative SEM investigation of the smear layer following preparation of root canals using nickel titanium rotary and hand instruments. J Oral Sci 49, 47-52.

17. Zarrabi MH, Bidar M, Jafarzadeh H (2006) An in vitro comparative study of apically extruded debris resulting from conventional and three rotary (Profile, Race, FlexMaster) instrumentation techniques. J Oral Sci 48, 85-88.

18. Schneider SW (1971) A comparison of canal preparations in straight and curved root canals. Oral Surg Oral Med Oral Pathol 32, 271-275.

19. Hülsmann M, Rümmelin C, Schäfers F (1997) Root canal cleanliness after preparation with different endodontic handpieces and hand instruments: a comparative SEM investigation. J Endod 23, 301306.

20. Versümer J, Hülsmann M, Schafers F (2002) A comparative study of root canal preparation using ProFile .04 and Lightspeed rotary Ni-Ti instruments. Int Endod J 35, 37-46.

21. Jeon IS, Spångberg LS, Yoon TC, Kazemi RB, Kum KY (2003) Smear layer production by 3 rotary reamers with different cutting blade designs in straight root canals: a scanning electron microscopic study. Oral Surg Oral Med Oral Pathol Oral Radiol Endod 96, 601-607. 